\title{
STRUCTURAL AND MAGNETIC PROPERTIES OF MnAs NANOCLUSTERS FORMED BY Mn ION IMPLANTATION IN
}

\section{GaAs}

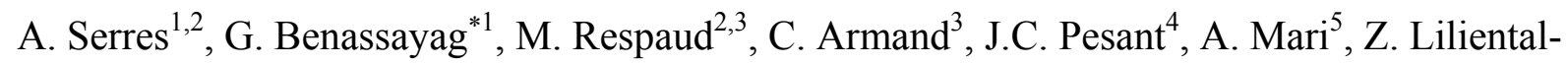 Weber $^{6}$ and A. Claverie ${ }^{1}$

\footnotetext{
${ }^{1}$ CEMES-CNRS, 29 rue J. Marvig BP 4347, 31055 Toulouse Cedex 4, France

${ }^{2}$ LPMC, INSA, 135 avenue de Rangueil, 31077 Toulouse, France.

${ }^{3}$ LNCMP, 143 avenue de Rangueil BP 4245, 31432 Toulouse, France.

${ }^{4}$ IEMN-CNRS, avenue Poincaré BP 69, 59652 Villeneuve d'Ascq Cedex, France

${ }^{5}$ LCC-CNRS, 205 Route de Narbonne, 31077 Toulouse, France.

${ }^{6}$ Lawrence Berkeley National Laboratory, Berkeley CA, 94720 m/s 62/203, US
}

\footnotetext{
*Corresponding Author: Tel. +33 56225 7900; E-mail: benassay@cemes.fr
} 


\section{Abstract}

Ferromagnetic (FM) nanostructures embedded in semiconductors are of fundamental interest since their physical properties could be used in new devices such as memories, sensors or spintronics.

In this work, we present results obtained on the synthesis and characterization of nanosized MnAs ferromagnets buried in GaAs. These nanocrystals are formed either by single Mn implantation or $\mathrm{Mn}+\mathrm{As}$ co-implantation at room temperature into GaAs wafers at 141 and $180 \mathrm{keV}$ respectively. Two doses, $1 \times 10^{16}$ and $2 \times 10^{16}$ ions. $\mathrm{cm}^{-2}$ for each impurity, are tested. Pieces of the wafers are then annealed by RTA or classical furnace annealing at various temperatures under $\mathrm{N}_{2}$ atmosphere for increasing times.

HRTEM and diffraction analysis show that under such conditions MnAs precipitates form with a regular hexagonal structure, the $3 \mathrm{~m}$ orientation-relationship of precipitates with respect to the matrix offers the most energetically stable configuration. Size distributions are systematically extracted from statistical analysis of "2 beam" TEM images. The precipitate mean diameters of nanocrystals populations range from 9 to $13 \mathrm{~nm}$ depending on the annealing conditions.

Magnetization measurements by SQUID magnetometry on the same samples reveal a progressive transition from a superparamagnetic behavior at room temperature to an FM one at $2 \mathrm{~K}$, reflecting a distribution of blocking temperature, due to distribution of size and to dipolar interactions. Curie temperatures in the range of $360 \mathrm{~K}$ were measured. 


\section{Introduction}

New hybrid magnetic semiconductor materials with low dimensions are of great fundamental and technological interest, as both the charge and the spin of the carriers can be used, which opens new fields in spintronics [1]. New devices such as memories, sensors, for example, may be developed by taking advantage of these new physical properties and their small size. Among the different possibilities to achieve ferromagnetic (FM) semi-conductors, the incorporation of Manganese in a GaAs substrate is one of the most promising. Most of the current studies deal with Molecular Beam Epitaxy to fabricate (Ga,Mn)As diluted phases [1]. These systems display Curie temperature $\left(T_{\mathrm{C}}\right)$ in the range of $80-110 \mathrm{~K}$, therefore limiting their potential application. Higher $T_{\mathrm{C}}$ can be reached by forming pure MnAs phases. Nanoclusters (ncs) can be formed also by means of MBE [2-3]. However, Mn ion implantation in GaAs followed by heat treatment is another route to synthesize nanocluster populations of MnAs embedded in a GaAs matrix [3-7]. The advantages of this method are the accurate control of the depth positioning of ncs through the energy of the ions, the density of ncs by adjusting the dose and the mean size by tuning the thermal treatment. In this work, we present a full analysis of the effects of the synthesis conditions on nanosized MnAs ferromagnets characteristics fabricated by Mn ion implantation. The Manganese profiles are followed by Secondary Ion Mass Spectrometry (SIMS) on as-implanted and annealed samples for various doses, annealing temperatures and times. We show the effect of the Mn diffusivity on the final material. The impact of co-implantation is analyzed. The crystalline structure and the magnetic properties are obtained respectively by Transmission Electron Microscopy (TEM) and SQUID magnetometry. Finally we propose two routes for a successful synthesis of this material. 


\section{Experimental details}

In order to fabricate MnAs nanomagnets, the goal is to introduce from 2 to $4 \%$ of $\mathrm{Mn}$ in the GaAs crystals which corresponds to doses in the $10^{16}$ ions. $\mathrm{cm}^{-2}$ range. We have studied two different conditions of implantation in semi-insulating (001) GaAs wafers. In the first case, we performed a co-implantation of $\mathrm{Mn}$ and As ions at energies of $141 \mathrm{keV}$ and $180 \mathrm{keV}$ respectively. These energies were chosen from a TRIM calculation in order to get the same depth for both Mn and As maximum concentrations, and to keep good stoichiometry along the $\mathrm{Mn}$ and As concentration profiles. The calculated projected range (Rp) corresponds to $77 \mathrm{~nm}$. The second case is a single Mn ion implantation at $141 \mathrm{keV}$. For both cases, two doses have been investigated : $1 \times 10^{16}$ and $2 \times 10^{16}$ ions.cm ${ }^{-2}$, which correspond respectively to a supersaturation of Mn of about $2 \%$ and $4 \%$.

Subsequently, implanted samples were annealed in a rapid thermal annealing (RTA) system, at various temperatures ranging from 600 to $850^{\circ} \mathrm{C}$ for several durations from 15 to 120 seconds under a $\mathrm{N}_{2}$ atmosphere and using a face-to-face method to avoid surface degradations. This thermal treatment induces several phenomena necessary for a proper synthezis of nanomagnets such as the solid phase epitaxy (SPE) of the GaAs matrix amorphized by ion implantation, the diffusion and nucleation to form MnAs precipitates and finally the growth of nanoclusters.

Several characterization methods have been used to study the material. The chemical composition and the distribution of Mn during annealing were investigated by Secondary Ion Mass Spectroscopy (SIMS) on an IMS 4/6F CAMECA analyzer. The structure of the nanoclusters was studied by conventional and High Resolution Transmission Electron Microscopy (TEM) on CM20 and CM30 Philips microscopes, respectively. Finally the 
magnetization of the samples was measured with a Superconducting Quantum Interference Device (SQUID) MPMS-5S Quantum Design magnetometer.

\section{Results and discussion}

\section{1 Chemical analysis.}

SIMS measurements have been carried out on all samples to follow the different fabrication steps from as-implanted to annealed. Figure 1 shows the Mn concentration profiles for a single implantation of manganese at a dose of $2 \times 10^{16}$ ions. $\mathrm{cm}^{-2}$ and for a co-implantation of $\mathrm{Mn}+\mathrm{As}$, at a dose of $2 \times 10^{16}$ ions. $\mathrm{cm}^{-2}$. The RTA annealing conditions were $750^{\circ} \mathrm{C}$ for $1 \mathrm{~min}$ in both cases. The as-implanted profiles (dotted line) are identical within experimental precision. In this profile, the projected range Rp is about $78 \mathrm{~nm}$ in good agreement with the TRIM simulations. At a depth of $150 \mathrm{~nm}$, a shoulder is observed which corresponds to the crystal/amorphous interface. The profile corresponding to the single implantation exhibits a large diffusion of Mn toward the surface and into the depth of the matrix. Around Rp, a large fraction of the Manganese atoms are lost. This Manganese has either been oxidized on the surface, as has been shown by TEM where a $\mathrm{MnO}_{2}$ phase was detected, or segregated at the End of Range (EOR) defects at roughly $150 \mathrm{~nm}$ below the surface where a secondary peak is present in the SIMS profile. On the contrary in the co-implanted profile it can be seen that most of the Mn remains close to the initial projected range of the as-implanted sample. In this case the loss of manganese around $\mathrm{Rp}$ is small. At the lower dose of $1 \times 10^{16}$ ions.cm ${ }^{-2}$ and for the same annealing conditions, for both single and co-implantation, Mn concentration profiles exhibit a large diffusion of $\mathrm{Mn}$. These results suggest that in a $\mathrm{Mn}+\mathrm{As}$ co-implantation process, high doses are necessary to preferentially form MnAs nanoclusters.

In figure 2 we have plotted the Mn concentration profiles for different RTA annealing temperatures ranging from $600^{\circ} \mathrm{C}$ to $850^{\circ} \mathrm{C}$ for one minute. This graph corresponds to a co- 
implantation at a dose of $1 \times 10^{16}$ ions.cm $\mathrm{cm}^{-2}$. It is clear that at $600^{\circ} \mathrm{C}$ the Manganese remains around the $\mathrm{Rp}=78 \mathrm{~nm}$, while for higher temperatures $\left(650^{\circ} \mathrm{C}\right.$ and $\left.850^{\circ} \mathrm{C}\right)$ the Manganese diffuses rapidly towards the surface and the crystal/amorphous interface in a first step, and finally toward greater depth in the GaAs for high temperatures. It is noticeable in figure 3, that both $\mathrm{Mn}$ profiles remain the same for both rapid thermal annealing (RTA) at $600^{\circ} \mathrm{C}$ for 1 min or classical furnace annealing (CTA) at $600^{\circ} \mathrm{C}$ for $30 \mathrm{~min}$. From these SIMS studies we conclude that for high implantation doses, a co-implantation Mn+As is necessary to trap the manganese at its original location whatever the thermal annealing. For a lower dose, the temperature has to be reduced to $600{ }^{\circ} \mathrm{C}$ to have a significant amount of the manganese remaining around the Rp. From figure 2 and 3 it is clear that the diffusion process is activated at temperatures ranging between $600{ }^{\circ} \mathrm{C}$ and $650{ }^{\circ} \mathrm{C}$. This could be put in relationship with the congruent temperature of $\mathrm{GaAs}\left(\mathrm{T}_{\mathrm{G}}=630^{\circ} \mathrm{C}\right)$ [8] when Arsenic begins to evaporate, which leads to a large injection of vacancies in the crystal.

\section{2 Structural properties.}

The same set of samples were observed by cross-sectional TEM images and by HRTEM. Figure 4(a) shows a High Resolution TEM image of one precipitate fabricated by a co-implantation at a dose of $2 \times 10^{16}$ ions. $\mathrm{cm}^{-2}$ and annealed at $750^{\circ} \mathrm{C}$ for $1 \mathrm{~min}$. A large precipitate with a diameter of about $9 \mathrm{~nm}$ is imaged. In this case, the crystal structure is hexagonal with characteristic lattice parameters $\mathrm{a}=0.37 \pm 0.01 \mathrm{~nm}$ and $\mathrm{c}=0.57 \pm 0.01 \mathrm{~nm}$ compatible with a MnAs composition. These values correspond to the well-known stable hexagonal phase MnAs. Most of the time, the orientation relationship between the matrix and the precipitates is $(0002)_{\mathrm{MnAs}} / /(\overline{111})_{\mathrm{GaAs}}$ and $[1 \overline{2} 10]_{\mathrm{MnAs}} / /[011]_{\mathrm{GaAs}}$. The observed orientation relationship (OR) is $3 m$ which provides the minimum interfacial and elastic energies for the precipitate/matrix. There are four variants of this OR. 
Figure $4(\mathrm{~b})$ is a cross-section image obtained on a co-implanted sample at a dose of $2 \times 10^{16}$ ion. $\mathrm{cm}^{-2}$ after $\mathrm{RTA}$ annealing at $750^{\circ} \mathrm{C}$ for $1 \mathrm{~min}$. In this picture, we can observe a good regrowth of the crystalline matrix and a population of nanoclusters at about $80 \mathrm{~nm}$ below the surface. Some extended defects, identified as End of Range (EOR) defects, are present at a depth of $150 \mathrm{~nm}$ which corresponds to the former crystal/amorphous interface. From a statistical analysis of the image, a mean diameter of about $12.8 \mathrm{~nm}$ was measured for the nanoclusters population in this case. TEM images have been taken for the single Mn implants and for lower doses with annealing temperature higher than $650^{\circ} \mathrm{C}$. No nanoclusters could be observed in these cases. However, on the images obtained on samples annealed at a temperature of $600{ }^{\circ} \mathrm{C}$, whatever the annealing time or method (RTA or CTA) used, nanoparticules were again found. These observations correspond perfectly with the SIMS profiles behavior described in the previous section. When the manganese remains at its original location either for the higher dose of the co-implanted samples or for an annealing temperature lower than $650^{\circ} \mathrm{C}$ nanoclusters are formed. In the other cases, the loss of manganese is too high and no clusters can be formed. If these conditions are respected, nanoclusters are formed with a broad size dispersion displaying an average diameter ranging from 9 to $13 \mathrm{~nm}$, depending on the annealing conditions.

\section{3 Magnetic properties.}

Magnetization measurements have been perfomed systematically on the different systems. Hysteresis loops have been measured at 2 and $300 \mathrm{~K}$, as well as the temperature dependence of the magnetization according to the zero field cooling procedures between 2 and $400 \mathrm{~K}$ in a field of $200 \mathrm{G}$. In most of the samples only the diamagnetic response of the GaAs substrate was evidenced. Interestingly only the samples containing MnAs ncs display a ferromagnetic signature. Figure 5(a) displays the extracted ferromagnetic response of the 
MnAs ncs in a high dose co-implanted sample $\left([\mathrm{Mn}]=210^{16} \mathrm{~cm}^{-2},[\mathrm{As}]=210^{16} \mathrm{~cm}^{-2}\right)$ annealed at $750^{\circ} \mathrm{C}$ for $1 \mathrm{~min}$. At $2 \mathrm{~K}$, the magnetization curves display a hysteristic behavior typical of a FM material. At room temperature, hysteresis disappears, which is characteristic of superparamagnetism. The superparamagnetic behavior [9] is confirmed by the zero field cooled - field cooled measurements (ZFC-FC) presented in Figure 5(b). We oberved a wide curve for the ZFC cycle which reflects a wide distribution of blocking temperatures $T_{B}$. This broadening of $\mathrm{T}_{\mathrm{B}}$ is probably due to both the size distribution of ncs and to dipolar interaction fields between nanoclusters. Curie temperature $\left(\mathrm{T}_{\mathrm{C}}\right)$ of this system was about $360 \mathrm{~K}$.

The different systems of MnAs ncs display the same behaviors. The relative high $\mathrm{T}_{\mathrm{C}}$ as compared to bulk MnAs phases $\left(\mathrm{T}_{\mathrm{C}}=318 \mathrm{~K}\right)$ can be ascribed to a small amount of $\mathrm{Ga}$ in the ncs. GaMn phases have large $\mathrm{T}_{\mathrm{C}}$ in the range of $600^{\circ} \mathrm{K}$. Ncs of $\mathrm{MnAs}_{1-\varepsilon} \mathrm{Ga}_{\varepsilon}$ with small $\varepsilon$ value should have lattice parameters near the MnAs since Ga and As have atomic radii in the same range, but with higher $\mathrm{T}_{\mathrm{C}}$, as previously observed by P.J. Wellman et al [6-7].

\section{Conclusion}

We have successfully formed MnAs nanosized ferromagnets in semi-insulating (001) GaAs by Mn ion implantation and subsequent annealing. The properties of such a system have been studied by SIMS, TEM and SQUID magnetometry. We have underlined the importance of the annealing process and particularly of the temperature on Mn diffusion. We have two different behaviors for temperature under and above the congruent temperature of GaAs, $\mathrm{T}_{\mathrm{G}}=633^{\circ} \mathrm{C}$. We have determined the position, the nature and size distributions of the nanoclusters by TEM. A mean diameter from 9 to $13 \mathrm{~nm}$ is observed. The ncs population exhibits a ferromagnetic behavior at low temperature. Superparamagnetic behavior is observed at higher temperature with a wide distribution of the blocking temperatures which reflects the size distribution of ncs size and dipolar fields. We also found a higher $\mathrm{T}_{\mathrm{C}}=360 \mathrm{~K}$ 
than the bulk possibly due to some Ga mixing in MnAs. This study has identified two ways to successfully synthesize MnAs nanoclusters using ion implantation.

\section{References:}

[1] H. Ohno, A. Shen, F. Matsukara, A. Oiwa, A. Endo, S. Katsumoto, Y. Iye, Appl. Phys. Lett. 363 (1996) 69

[2] J. De Boeck, R. Oesterholt, A. Van Hesch, H. Bender, C. Bruynseraede, C. Van Hoof, G. Borghs, Appl. Phys. Lett. 2744 (1996) 68

[3] J. De Boeck, R. Oesterholt, A. Van Hesch, H. Bender, C. Bruynseraede, C. Van Hoof, G. Borghs, J. Mag. Mag. Mat. 148 (1996) 156

[4] J. Shi, D. D. Awschalom, P. M. Petroff, K. Babcock, J. App. Phys. 4331 (1997) 81

[5] C. Chen, M. Cai, X. Wang, S. Xu, M. Zhang, X. Ding, Y. Sun, J. App. Phys. 5636 (2000) 87

[6] P. J. Wellmann, J. M. Garcia, J.-L. Feng, P. M. Petroff, Appl. Phys. Lett. 2532 (1997) 71

[7] P. J. Wellmann, J. M. Garcia, J.-L. Feng, P. M. Petroff, Appl. Phys. Lett. 3291 (1998) 73

[8] C. Chatillon, D. Chatain, J. Cryst. Growth 91 (1995) 151

[9] L. Néel, Ann. Geophys. 99 (1949) 5

Figure Captions:

Fig. 1. SIMS measurement of Manganese concentration of (Mn,As):GaAs and Mn:GaAs annealed at $750^{\circ} \mathrm{C}, 1 \mathrm{~min}\left([\mathrm{Mn}]=2.10^{16} \mathrm{~cm}^{-2} ;[\mathrm{As}]=2.10^{16} \mathrm{~cm}^{-2}\right)$. As-implanted profile is drawn as dotted line.

Fig. 2. SIMS measurement of Manganese concentration of (Mn,As):GaAs $\left([\mathrm{Mn}]=1.10^{16} \mathrm{~cm}^{-}\right.$ ${ }^{2} ;[\mathrm{As}]=1.10^{16} \mathrm{~cm}^{-2}$ ) annealed for $1 \mathrm{~min}$ at various temperatures. 
Fig. 3. SIMS measurement of Manganese concentration of (Mn,As):GaAs $\left([\mathrm{Mn}]=1.10^{16} \mathrm{~cm}^{-}\right.$ ${ }^{2} ;[\mathrm{As}]=1.10^{16} \mathrm{~cm}^{-2}$ ) annealed at $600^{\circ} \mathrm{C}$ for two different durations.

Fig. 4. (a) Cross-sectional TEM image ("2 beams", $g=(220))$ of (Mn,As):GaAs annealed at $750^{\circ} \mathrm{C}, 1 \mathrm{~min}\left([\mathrm{Mn}]=2 \cdot 10^{16} \mathrm{~cm}^{-2} ;[\mathrm{As}]=2 \cdot 10^{16} \mathrm{~cm}^{-2}\right)$. (b) High Resolution TEM image of a single MnAs nanoparticle of (Mn,As): GaAs annealed at $750^{\circ} \mathrm{C}, 1 \mathrm{~min}\left([\mathrm{Mn}]=2.10^{16} \mathrm{~cm}^{-2}\right.$; $\left.[\mathrm{As}]=2 \cdot 10^{16} \mathrm{~cm}^{-2}\right)$.

Fig. 5. Magnetization measurements of $(\mathrm{Mn}, \mathrm{As}): \mathrm{GaAs}$ annealed at $750^{\circ} \mathrm{C}, 1 \mathrm{~min}([\mathrm{Mn}]=$ $\left.2.10^{16} \mathrm{~cm}^{-2} ; \mathrm{As}\right]=2 \cdot 10^{16} \mathrm{~cm}^{-2}$ ). The diamagnetic response has been removed. (a) Hysteresis loops at room temperature and $\mathrm{T}=2 \mathrm{~K}$; (b) Zero field cooled - field cooled magnetization measurement for $\mathrm{H}=200 \mathrm{oe}$. 